\title{
Perspective on imaging services at the London 2012 Olympic and Paralympic Games
}

\author{
Sarath Bethapudi • Robert S. D. Campbell • \\ Philip O'Connor
}

Received: 14 March 2014 / Revised: 1 June 2014 / Accepted: 8 June 2014 / Published online: 2 July 2014

(C) ISS 2014

\section{Introduction}

The 2012 Olympic and Paralympic Games were widely acclaimed a success for host city London. Over 10,000 athletes from 196 countries participated in the Olympic Games, whilst the Paralympics witnessed over 4,000 athletes from 164 countries. The medical services at the games were offered through purpose-built polyclinics. Three such purpose-built polyclinics were set up, one each at the main games village in Stratford, the rowing village in Eton Dorney and sailing village at Weymouth. The Stratford Polyclinic was the major polyclinic within the main games village. Besides onsite consultation from sports physicians and general practitioners, fully equipped imaging department, accident and emergency, physiotherapy, podiatry, chiropody, dentistry, optometry and laboratory services were offered within the polyclinics. Accident and emergency services were operational $24 \mathrm{~h}$, and the rest of the polyclinic functioned from 7:00 a.m. in the morning to 11:00 p.m. in the evening, over two shifts. The polyclinics opened a week before the start of the games and continued to provide services for 2 days after the closing ceremonies, functioning for a total of 30 days for the Olympics and 21 days for the Paralympics.

A total of 7.9 million euros ( $£ 6.6$ million, $\$ 11$ million U.S) was invested into setting up the imaging department.

\section{S. Bethapudi}

County Durham \& Darlington NHS Trust, Durham, UK

R. S. D. Campbell

Royal Liverpool and Broadgreen University Hospitals NHS Trust, Liverpool, UK

P. O'Connor $(\square)$

Leeds Teaching Hospitals NHS Trust and the NIHR Leeds

Musculoskeletal Biomedical Research Unit, Leeds, UK

e-mail: philip.o'connor@leedsth.nhs.uk
The polyclinic at Stratford was equipped with a digital x-ray system (GE XR 656 wireless digital x-ray), two US machines (GE Logiq E9 scanners), a 64-slice multidetector CT with fluoroscopy facilities for intervention (GE Discovery 750 HD CT scanner), and 3- and 1.5-T wide-bore MRI scanners (GE Medical, Milwaukee, WI). The Eton Dorney and Weymouth Polyclinics were equipped with US scanners (GE Logiq E9 scanners). Integrated GE RIS-PACS with dual monitor work stations and voice recognition facilities were used for studying and reporting the examinations performed. Conference facilities separate from the main reporting room with a high-definition projector were set up to discuss the scan images with the team doctors in a confidential environment. All examinations were printed, and compact discs were issued along with a copy of the radiologist's report to the athletes scanned straight after the examination. Both electronic and hand-filled request cards were in use, and referrals were received from team doctors, doctors consulting within the polyclinic and venue medical doctors. Twenty-seven musculoskeletal (MSK) radiologists, 56 radiographers, 23 radiographic assistants and 4 ultrasonographers offered their services for the Olympic Games. Twelve MSK radiologists, ten radiographers, five radiographic assistants and two trained ultrasonographers manned the services during the Paralympics. During the competitions' busiest schedule, five radiologists worked each shift during the Olympics and two radiologists per shift for the Paralympics at Stratford Polyclinic. At any given time, one radiologist was based at Eton Dorney and Weymouth Polyclinics during functional hours. This facilitated hot reporting, and reports were generally issued within $1 \mathrm{~h}$ of performing the radiological investigations. Presence of onsite radiologists obviated the need for teleradiology services. Any imaging requests arising from casualty consultations outside hours were sent to regional hospitals, these were few in number. 
We were aware of the very limited data available from previous summer games. To collect detailed demographic data and to quantify the diagnostic and therapeutic impact of imaging at the games, a part industry-funded imaging fellow was appointed. This allowed more comprehensive data on imaging to be acquired and published [1,4]. We hope to convey important learning points from our experiences, which could be helpful in planning and setting up imaging services at future major international sporting events.

\section{Imaging at the London 2012 Olympics}

A total of 1,711 diagnostic investigations and interventional procedures were performed at Stratford Polyclinic, of which 835 (49 \%) were MRIs, 347 (20\%) diagnostic USs, 50 (3\%) CTs and $405(24 \%)$ x-ray examinations [1]. Seventy-four imaging-guided interventional procedures were performed, of which 45 were performed under US guidance and 29 were CT guided. Of all investigations, $75 \%$ were performed on athletes, $20 \%$ on team officials and $5 \%$ on the workforce. Track and field athletes accounted for $35 \%$ of all investigations performed. Sixty-five US examinations were performed at Eton Dorney and Weymouth [1].

US was the first line investigation for suspected superficial pathologies such as tendinopathy, tenosynovitis, tendon tears, bursitis and plantar fascial injury. US was also used to diagnose muscle tears, although some of these were subsequently investigated with MRI for better assessment of morphology and grading, as this impacted on the management plan. Radiologists performed all MSK US examinations and ultrasonographers performed non-MSK US examinations.

Sixty-four percent of all MRIs performed were on the lower extremities, and nearly one in four MRIs performed were knee examinations. MRIs on the spine were performed mostly for suspected stress fractures and intervertebral disc disease, constituting $19 \%$ of all MRIs performed [1]. Use of contrast was limited as most of the pathology was readily visualised on non-contrast studies. In total, contrast was used only in two cases throughout the competition, one of which was an incidental tumour of the abdominal wall and the other was an MRI arthrogram. Fifteen acute ligament injuries of the elbow joint were witnessed, 12 of which were ulnar collateral ligament injuries and the rest were combination injuries involving both medial and lateral ligaments [2]. The majority of elbow ligament injuries were witnessed in judo and weightlifting disciplines. This is an interesting observation as the majority of the current literature on elbow injuries in sports is based on throwing athletes, especially baseball pitchers. Acute plantar fascia and Achilles tendon injuries were seen in 12 athletes from track and field, handball, basketball and gymnastics [3].
Having a CT scanner on site proved extremely useful during the games. Athletes in whom MRI scans revealed bone marrow oedema consistent with a stress response subsequently underwent $\mathrm{CT}$ to establish the presence or absence of a fracture. This was particularly important in the context of the games, as it was a major factor influencing an athletes decision whether to compete or withdraw. Bone stress response and stress fractures were seen in nearly 50 athletes, mostly from track and field events and gymnastics. Interventional procedures were performed under both CT and US guidance. All spinal interventional procedures were exclusively performed under CT fluoroscopic guidance, and these included nerve root blocks, facet joint injections and spinal epidural blocks. The majority of US-guided interventional procedures performed were local anaesthetic and steroid injections. Prolotherapy (PRP) injections were also performed in smaller numbers during the competitions. Significant incidental findings included a tumour of the rectus abdominis muscle and liver lesions, both in team officials. Ovarian torsion was diagnosed on US in an athlete with acute lower abdominal pain. This was confirmed and operated upon on the same day following referral to an acute hospital. Most of the plain radiographs performed were for suspected acute bony injuries. Chest x-rays for suspected general illnesses were also performed in considerable numbers.

It was observed that pathology witnessed during each stage of the competition coincided with the scheduled events. Elbow injuries occurred more commonly during the days judo and weightlifting were scheduled, with up to six UCL injuries occurring in a single day. Hamstring, plantar fascia and Achilles tendon injuries coincided with track and field events. This changing pattern of injury was entirely predictable with huge differences in the pathologies seen day to day.

\section{Imaging at the Paralympics}

The infrastructure and imaging facilities were the same as those used for the Olympic Games. Six hundred fifty-five radiological investigations and procedures were performed in total, of which 637 were diagnostic examinations and 18 were interventional procedures. Of these, $254(39 \%)$ were MRIs, 216 were $\mathrm{x}$-rays (33\%), 157 were US (24\%) and 28 (4\%) were CTs. Nearly $65 \%$ of all radiological examinations were performed on athletes and the rest on team officials and the workforce [4].

Track and field and power-lifting athletes alone accounted for more than $25 \%$ of all investigations among athletes in the single discipline participation. Track and field/long jump and shot put/discus throw athletes were among the most frequently scanned in the multidisciplinary participation, accounting for 
nearly $10 \%$ of all investigations on athletes. Suspected acute bony injury, spinal pathology for back pain/sciatica, muscle tears and tendon pathology were among the common clinical indications, leading to the radiological investigations in over $50 \%$ of athletes. Within the athletes' category, nearly $20 \%$ of all diagnoses made on imaging were tendon pathologies, $10 \%$ muscle tears, $10 \%$ spinal pathologies, $6 \%$ acute fractures and $5 \%$ ligament injuries. Sixty percent of tendon pathologies involved the shoulder rotator cuff tendons and close to $90 \%$ of the muscle tears were in the lower limbs. The majority of the ligament injuries diagnosed on imaging were in the ankle joint [4]. Most of the imaging performed during the Paralympics was for suspected upper limb injuries in athletes in contrast to the able-bodied athletes scanned during the Olympic Games, where the majority of radiological investigations performed were for suspected lower limb injuries.

Having wide-bore MR magnets was extremely helpful during the Paralympics as this offered extra room and flexibility in positioning the disabled athletes with functional limitation in movement and chronic contractures. CT whole-body scanograms were performed in a few cases in preference to radiographs to exclude the presence of metallic foreign bodies, implants, etc., where there was uncertainty regarding MR safety and compatibility. CT was used as the primary investigation for trauma in some Paralympic athletes with complex limb deformities. In retrospect, it would have been useful to have access to conscious sedation for interventional procedures in some Paralympic athletes, where involuntary muscle spasms can limit access, particularly in CT-guided procedures.

\section{Conclusion}

The spectrum and nature of injuries seen at the London 2012 Olympic and Paralympic Games were generally comparable, although the majority of injuries witnessed at the Paralympics were in the upper extremity, whilst those at the Olympic Games were mostly in the lower extremities. Track and field athletes were the most commonly imaged at the 2012 games. MRI was the most widely used radiological investigation and the CT scanner was the least utilised during both the Olympic and Paralympic Games. Having two MRI scanners at the main games village polyclinic was immensely helpful. The widebore nature of the magnets was helpful with the scanner fully active 16 hours a day throughout the games in the context of scanning disabled athletes during the Paralympic Games.

Our aim at the Olympics and Paralympics was to deliver one of the best radiology services the summer games had ever experienced. This required substantial advanced planning and work on setting up imaging services began in 2007. Changes to budgets and delivery dates required adjustment of plans right up to games time. One of our major issues was leave and training. All staff delivering the games radiology service were volunteers providing a minimum of 10 days service using their own free time to support the Olympics without expenses for accommodation or travel, which reduced volunteer costs. To work as a volunteer also required 2 days generic and 1 day role-specific training for the London organising committee of the Olympic Games. Given an average annual leave entitlement of 30 days per year for medical staff in the UK, each volunteer spent nearly $43 \%$ of their 2012 holidays offering their services at the games. Only a days' training was allocated to learn all the radiology systems required. This was especially problematic for MR scanning with many radiographers unfamiliar with the MR scanners being used. We got around this by using training onsite via existing staff and applications specialists during the first days of any volunteering period.

The experience of developing and delivering a Summer Olympics and Paralympics service was exhausting and at the same time exceptionally rewarding. We strongly recommend it to future summer games radiologists.

Acknowledgments The authors would like to thank the entire radiology volunteer workforce at the Olympic and Paralympic Games for successful delivery of imaging services and GE and Leeds Teaching Hospitals NHS Trust for funding an imaging fellowship during the games.

Conflict of interest No conflict of interest.

\section{References}

1. Bethapudi S, Budgett R, Engebretsen L, O'Connor P. Imaging at London 2012 summer Olympic Games: analysis of demand and distribution of workload. Br J Sports Med. 2013;47(13):850-6.

2. Bethapudi S, Robinson P, Engebretsen L, Budgett R, Vanhegan IS, O'Connor P. Elbow injuries at the London 2012 Summer Olympic Games: demographics and pictorial imaging review. AJR Am J Roentgenol. 2013;201(3):535-49.

3. Elias DA, Carne A, Bethapudi S, Engebretsen L, Budgett R, O'Connor P. Imaging of plantar fascia and Achilles injuries undertaken at the London 2012 Olympics. Skelet Radiol. 2013;42(12):1645-55.

4. Bethapudi S, Campbell RSD, Budgett R, Willick SE, Van De Vliet P. Data analysis of imaging services at the paralympic games London 2012. Br J Sports Med. 2014. doi:10.1136/bjsports-2013093386. 